\title{
Testing Rhodiola sachalinensis saccharide as cryoprotectant for bovine spermatozoa
}

\author{
Hualin Cao, ${ }^{* 1}$ Xiuzhu Sun, ${ }^{* 1}$ Qingwang Li, ${ } \dagger^{2}$ Shengxian Zhou, ${ }^{*}$ Xiangbin Nan, ${ }^{*}$ Jianhong Hu, ${ }^{*}$ Liqiang Wang, ${ }^{*}$ \\ and Yamei He* \\ ${ }^{*}$ College of Animal Science and Technology, Northwest A\&F University, Yangling, Shaanxi 712100, China \\ †College of Environmental and Chemical Engineering, Yanshan University, Qinhuangdao, Hebei 066004, China
}

\begin{abstract}
Rhodiola sachalinensis saccharide (RSS) was extracted from the rhizome of Herba Rhodiolae and was expected as a novel cryoprotectant. The aim of this study was to test the effects of RSS on motility of bull sperm and the activities of superoxide dismutase (SOD), lactate dehydrogenase (LDH), and glutamic oxaloacetic transaminase (GOT) in bull sperm during cryopreservation. Rhodiola sachalinensis saccharide was added at the concentrations of $0.02,0.04,0.06,0.08$, and 0.10 $\mathrm{mg} / \mathrm{mL}$ to the extenders, which were used to store bovine semen. It was found that the RSS-added extends resulted in a higher percentage of cryopreserved sperm motility, mitochondrial activity, and membrane and acrosome integrity than those of RSS-free extenders. The SOD, LDH, and GOT activities were all decreased during the process of freezing and thawing. The extenders supplemented with RSS improved the SOD, LDH, and GOT activities after cryopreservation compared with the RSS-free groups. In conclusion, RSS conferred great cryoprotective capacity to the basic extender for bull spermatozoa during the process of freezing-thawing, and the optimal concentration of RSS for the extender was $0.06 \mathrm{mg} / \mathrm{mL}$.
\end{abstract}

Key words: bull semen, cryopreservation, Rhodiola sachalinensis saccharide, superoxide dismutase

\section{INTRODUCTION}

The cryopreservation of spermatozoa developed with wide use for AI. It aims at extending the life of sperm in vitro by reducing their metabolism. Highquality male animals could be used to enlarge herds quickly and improve production of offspring by AI with frozen-thawed sperm because no limits of time and region exist for this technology. However, lower fertility

\footnotetext{
Received January 25, 2013.

Accepted June 29, 2013.

${ }^{1}$ Authors Hualin Cao and Xiuzhu Sun contributed equally to this

${ }^{2}$ Corresponding author: ysulqw@126.com
} work. has been shown in bovines, swine, and sheep because of lower motility of frozen-thawed sperm (Parrish et al., 1995; Maxwell et al., 1999; Eriksson et al., 2002). During the process of freeze-thawing, sperm are easily affected by many factors, including diluents, temperature, conditions of freeze-thawing, and especially by cryoprotectants (Johnson et al., 2000; Schäfer-Somi et al., 2006). Different kinds of cryoprotectants have been added into extenders of semen to try to reduce the damage to sperm during the process of freeze-thawing and to keep sperm motility. Based on whether they get into cells, cryoprotectants are divided into 2 types, one of which is permeating cryoprotectants, including glycerol, ethylene glycol, dimethyl acetamide, and dimethyl sulfoxide, which are able to permeate through cell membranes and prevent cells from freeze-thaw injury (Li et al., 2005). Glycerol was once regarded as an effective cryoprotectant for mammalian sperm and applied to AI (Kumar et al., 2003), but it cannot be used with high concentration due to its toxicity to sperm (Garner, 1991; Katkov et al., 1998). Another type of cryoprotectant is non-permeating cryoprotectants (such as saccharides), which improve the extracellular osmotic pressure because they cannot enter cells. They stimulate moisture to exude out the cells, cause cellular dehydration and shrinkage, reduce the amount of intracellular ice crystal formation, and ultimately protect cells from damage (Gonda and Sei, 2005). Therefore, it would be better to try to find other materials that can preserve sperm motility during the freeze-thaw process. Several papers have been published in the last few years that involved utilization of saccharides in frozen semen, and have shown that lactose, saccharose, and polysaccharides all have some function in the protection of frozen-thawed sperm of bulls, rams, and boars (Liu et al., 1998; Salamon and Maxwell, 2000; Corcuera et al., 2007; Gómez-Fernández et al., 2012). Fructose-based extenders yielded a higher post-thaw sperm motility and viability than did sorbitol-based extenders (Chanapiwat et al., 2012). Trehalose was reported to be added as a cryoprotectant into the extender of frozen-thawed sperm and an obvious increase 
of sperm motility was found in cryoprotected sperm of bulls (Woelders et al., 1997), mice (Sztein et al., 2001), and goats (Aboagla and Terada, 2004). Furthermore, plant polysaccharides have been shown some positive effects of oxidation resistance on frozen-thawed sperm of domestic animals, based on the data published in recent years (Hu et al., 2009).

Herba Rhodiolae is a traditional Chinese medicine with strong vitality and particularly adaptability, and Rhodiola extract helps to increase RNA content in skeletal muscle, promotes ATP resynthesis, changes the brain neurotransmitter transfer pathway, and improves people's physical and mental energy (Abidov et al., 2003; Zhang et al., 2007). Rhodiola sachalinensis saccharide (RSS), extracted from Herba Rhodiolae, is a plant polysaccharides composed of rhamnose, Arab sugar, xylose, mannose, glucose, galactose, and galacturonic acid (Song et al., 2008). Rhodiola sachalinensis saccharide acts as an important role of strong oxidation resistance, anti-aging and apoptosis prevention, and possesses highly antiinflammatory properties (Yousef et al., 2006). In this study, RSS was added into the extenders of bovine semen during the freeze-thaw process and because of the characteristics of oxidation resistance in humans, we tried to find some information on RSS in cryopreserved sperm.

\section{MATERIALS AND METHODS}

\section{Chemical Agents}

All chemicals used in the present experiments were purchased from Sigma Chemical Co. (St. Louis, MO), unless otherwise stated.

\section{The Extraction of RSS}

Rhodiola sachalinensis saccharide was extracted from the rhizome of Herba Rhodiolae by the ethanol precipitation method from the experiment of Gong et al. (2005). The dried rhizome of the Herba Rhodiolae was soaked for $5 \mathrm{~h}$ in distilled water (held at $80^{\circ} \mathrm{C}$ ) after being smashed and sifted. The extracting solution was filtrated 5 times and concentrated using a rotary evaporator (Shanghai Jinghe Analytical Apparatus Co. Ltd., Shanghai, China). After filtering, one-third volume of chloroform: $n$-butyl alcohol (4:1) was added, and the mixture was shaken for $30 \mathrm{~min}$. Then, it was centrifuged at 4,000 $\times g$ for $30 \mathrm{~min} ; 85 \%$ ethanol was added to the solution $(3: 1, \mathrm{vol} / \mathrm{vol})$ and left $12 \mathrm{~h}$. After it was centrifuged at $4,000 \times g$ for $30 \mathrm{~min}$, the sediment was collected and left for about $12 \mathrm{~h}$ and dried by vacuum freeze dryer (Shanghai Tofflon Science and Technology Co. Ltd., Shanghai, China) for more than $24 \mathrm{~h}$ to produce the RSS. The powdery RSS was dissolved in double-distilled water to get the RSS liquid supplement, which was added to the Tris-citric acid-glucose (TCG) extender to obtain the specific concentration of RSS in this study. The net content of the RSS liquid supplement was $0.20 \mathrm{mg} / \mathrm{mL}$.

\section{Semen Collection}

Semen was collected by artificial vagina from 6 mature Holstein bulls (aged 4 to 5 yr) at the Domestic Animal Improving Station (Shaanxi Province, China) and 2 ejaculates were obtained from each bull. After the semen samples were evaluated for volume, sperm concentration, and percentage of motile spermatozoa, bull ejaculates with $>75 \%$ progressive motility and $>85 \%$ normal sperm morphology before cryopreservation were used in this study. In total, 12 ejaculates from 6 bulls were used and each ejaculate was apportioned between the treatments and control.

\section{Diluent Preparation}

In this study, the diluents were prepared according to the method of Hu et al. (2006). The basic diluent (TCG) consisted of $1.1 \mathrm{~g}$ of glucose, $1.48 \mathrm{~g}$ of citric acid, and $2.42 \mathrm{~g}$ of Tris dissolved in $100 \mathrm{~mL}$ of deionized water. The freezing extender of the control was composed of basic diluent TCG plus $25 \mathrm{mg}$ of gentamicin, 50,000 IU of penicillin, and $20 \mathrm{~mL}$ of egg yolk per $100 \mathrm{~mL}$ of sterile nonpyrogenic water. For the basic freezing diluent for the treatment, TCG was mixed with different concentrations of RSS $(0.02,0.04,0.06,0.08$, and 0.10 $\mathrm{mg} / \mathrm{mL}$ ). The cooling extender was composed of $25 \mathrm{mg}$ of gentamicin, 50,000 IU of penicillin and $20 \mathrm{~mL}$ of egg yolk per $100 \mathrm{~mL}$ of basic freezing diluent. The freezing extenders of the control and treatments were composed of the cooling extender and $3 \%$ (vol/vol) glycerol, respectively.

\section{Semen Freezing}

The fresh semen was divided into 6 equal fractions. One fraction was diluted with the control extender and the others with the treatment extender (supplemented with $0.02,0.04,0.06,0.08$, and $0.10 \mathrm{mg} / \mathrm{mL}$ of RSS) to obtain $1.0 \times 10^{9} \mathrm{sperm} / \mathrm{mL}$. Semen was cooled from 37 to $4^{\circ} \mathrm{C}$ and allowed to equilibrate for $1.5 \mathrm{~h}$. Polyvinyl chloride straws $(0.25 \mathrm{~mL}$; IMV Technologies France, L'Aigle, France) were filled and maintained at $4^{\circ} \mathrm{C}$ for 2.5 $\mathrm{h}$. These straws were then placed about $3 \mathrm{~cm}$ above the liquid nitrogen surface where the temperature was approximately $-120^{\circ} \mathrm{C}$. After $10 \mathrm{~min}$, they were immersed directly into the liquid nitrogen $\left(-196^{\circ} \mathrm{C}\right)$ for storage. 


\section{Semen Thawing}

Thawing was performed in a water bath at $37^{\circ} \mathrm{C}$ for $45 \mathrm{~s}$. Subsequently, the sperm parameters of all sperm samples were evaluated.

\section{Analysis of Sperm Motility}

Sperm motility was assessed by determining the percentage of spermatozoa showing any movement of the flagellum. The percentage of linear motile sperm was examined visually by computer-assisted sperm analysis systems (WLJY-9000; WeiLi Software Co. Ltd., Beijing, China). Each sperm sample was thawed $45 \mathrm{~s}$ at $37^{\circ} \mathrm{C}$ and incubated $10 \mathrm{~min}$ at $37^{\circ} \mathrm{C}$. Five microliters of each straw was examined to determine the mobility parameters of 100 to 150 sperm in 5 random microscopic fields. The mobility parameters included the percentage of motile sperm, the curvilinear line velocity (VCL; $\mu \mathrm{m} / \mathrm{s})$, the straight line velocity $(\mathbf{V S L} ; \mu \mathrm{m} / \mathrm{s})$, linearity index (linearity index $=$ VCL/VSL $\times 100 \%$ ), and amplitude of the lateral head displacement (ALH).

\section{Analysis of Membrane Integrity}

The sperm membrane integrity was evaluated by the hypoosmotic swelling test after the process of freezethawing (Osinowo et al., 1982). The $50 \mu \mathrm{L}$ of seminal fluid was diluted with $1 \mathrm{~mL}$ of hypoosmotic solution (7.35 g of sodium citrate $2 \mathrm{H}_{2} \mathrm{O}$ and $13.51 \mathrm{~g}$ of fructose in $1 \mathrm{~L}$ of distilled water) and incubated for $30 \mathrm{~min}$ at $37^{\circ} \mathrm{C}$ (Jeyendran et al., 1984). Semen samples were observed after the hypoosmotic swelling test under light microscopy at $400 \times$ magnification. The percentage of spermatozoa with coiled tails was calculated. At least 200 spermatozoa per slide were observed.

\section{Analysis of Acrosome Integrity}

Acrosome integrity was assessed by the fluorescein isothiocyanate-conjugated peanut agglutinin (FITCPNA) method (100 $\mu \mathrm{g} / \mathrm{mL}$; Aboagla and Terada, 2003). The thawed semen tubules were put into $2 \mathrm{~mL}$ of $3 \%$ polyvinylpyrrolidone fluid (the solution was prepared by mixing $3 \mathrm{~g}$ of polyvinylpyrrolidone in $100 \mathrm{~mL}$ of PBS) and centrifuged 2 times at $800 \times g$ for $3 \mathrm{~min}$. After the removal of the supernatant, the pellet was suspended with PBS buffer and $30 \mu \mathrm{L}$ was used to make smears. After air drying, sperm smears were fixed with pure methanol for $10 \mathrm{~min}$. About 30 $\mu \mathrm{L}$ of FITC-PNA solution in PBS was spread over a microscope slide. The slides were then incubated in a dark and moist chamber for $30 \mathrm{~min}$ at $37^{\circ} \mathrm{C}$. After incubation, the slides were rinsed with PBS, air dried, and mounted with $10 \mu \mathrm{L}$ of the antifade solution to preserve fluorescence. The slide smear was covered by a coverslip and sealed with colorless nail polish. The acrosome status of the sperm was examined by an epifluorescence microscope (Leika DM-IRB; Leica Microsystems, Wetzlar, Germany) linked to a Nikon digital camera DXM (Nikon Ltd., Tokyo, Japan) with 480-nm excitation and 530-nm emission settings. The whole acrosome was visualized with strong green fluorescence under the epifluorescence microscope and was scored as acrosome-intact sperm cells. The percentage of fluorescent acrosome-intact spermatozoa was determined from at least 200 sperm cells per slide.

\section{Analysis of Mitochondrial Activity}

Rhodamine 123 staining was used to estimate the mitochondrial activity of sperm (Garner et al., 1997). The thawed sperm were stained by incubation at $37^{\circ} \mathrm{C}$ in the dark for $15 \mathrm{~min}$ in $1 \mu \mathrm{g} / \mathrm{mL}$ of rhodamine 123 and then counted under a fluorescence microscope (COIC TBE 2000; Chongqing Optical Co. Ltd., Chongqing, China) at $400 \times$ magnification. Sperm showing green fluorescence in the midpiece were considered to be positive for mitochondrial activity. At least 200 spermatozoa were observed per slide.

\section{Biochemical Assay}

After collection and the process of freeze-thawing, biochemical assays were performed on fresh sperm and frozen-thawed sperm samples immediately. The volumes of $120 \mu \mathrm{L}$ of fresh sperm and frozen-thawed sperm samples were centrifuged at $1,600 \times g$ for 5 min at $25^{\circ} \mathrm{C}$. After elimination of the supernatant, 360 $\mu \mathrm{L}$ of Triton X-100 (1\%, vol/vol) was added into the precipitate to extract for $20 \mathrm{~min}$, and centrifuged at $4,000 \times g$ for $30 \mathrm{~min}$ at $25^{\circ} \mathrm{C}(\mathrm{Hu}$ et al., 2009). The supernatants were collected as the crude extracts of enzymes in sperm. The enzymatic activities of superoxide dismutase (SOD), lactate dehydrogenase (LDH) and glutamic oxaloacetic transaminase (GOT) were measured and all expressed as units per milliliter.

SOD Assay. The SOD activity was analyzed using the technique of Flohé and Otting (1984) at 560 $\mathrm{nm}$. Each sperm sample was diluted 1:5 with PBS (50 $\mathrm{m} M, \mathrm{pH}$ 7.0). The assay solution contained sodiumcarbonate buffer $(50 \mathrm{~m} M, \mathrm{pH} 10.0), 0.1 \mathrm{~m} M$ xanthine, $0.025 \mathrm{~m} M$ nitro blue tetrazolium, $0.1 \mathrm{~m} M$ EDTA, and xanthine oxidase, and samples were mixed in a small cuvette. One unit of SOD activity was defined as the amount of enzyme required to cause $50 \%$ inhibition of the rate of nitro blue tetrazolium chloride reduction. The activities of SOD were detected using a kit (Nan- 
jing Jiancheng Bioengineering Institute, Nanjing, China) and measured on the spectrophotometer (Shanghai Spectrophotometer Co. Ltd., Shanghai, China).

$\boldsymbol{L D H} \boldsymbol{A s s a y}$. The LDH activity was determined at $37^{\circ} \mathrm{C}$ based on the characteristic absorption of NADH at $340 \mathrm{~nm}$ (Blanco et al., 1976). One unit of enzyme activity was considered to be the amount producing an optical density change of $2.07 / \mathrm{min}$ at $340 \mathrm{~nm}$, which corresponds to oxidation of $1 \mu \mathrm{mol}$ of $\mathrm{NADH}$ in the conditions of the assay. The enzymatic activities of $\mathrm{LDH}$ were detected using a kit (Biosino Bio-Technology and Science Inc., Beijing, China) on the spectrophotometer (Shanghai Spectrophotometer Co. Ltd.).

GOT Assay. The GOT activity was measured at $25^{\circ} \mathrm{C}$ using a method based on the fact that GOT catalyzed the transfer of the L-aspartate amino group to oxoglutarate, observed with the spectrophotometer (Shanghai Spectrophotometer Co. Ltd.) at $340 \mathrm{~nm}$ (Borque and Ayllón, 1996). One unit of GOT activity was equivalent to a change in optical density of $0.001 /$ min at $340 \mathrm{~nm}$. The enzymatic activities of GOT were tested using a kit (Nanjing Jiancheng Bioengineering Institute).

\section{Statistical Analysis}

All results were expressed as mean values \pm standard error of the mean. The mean values of the percentages of motile sperm, acrosome-intact sperm, plasma membrane-intact sperm, and mitochondrial activity of spermatozoa were compared using Duncan's multiple range test using ANOVA when the $F$-value was significant $(P<0.05)$. All analyses were performed by using SPSS software (SPSS 11.5 for Windows; SPSS Inc., Chicago, IL).

\section{RESULTS}

\section{The Characteristics of Sperm Motion}

The motility of frozen-thawed sperm supplemented with 0.04 and $0.06 \mathrm{mg} / \mathrm{mL}$ of RSS was significantly higher than that of other concentrations $(P<0.05)$, but no significant difference existed between them (Table 1). The VCL value of sperm containing 0.06 and $0.08 \mathrm{mg} / \mathrm{mL}$ of RSS in extenders was higher than that of in other groups $(P<0.05)$. For 0.04 and $0.06 \mathrm{mg} /$ $\mathrm{mL}$ of RSS in the diluents, the sperm ALH value was significantly higher than the other diluents $(P<0.05)$. The extender with $0.06 \mathrm{mg} / \mathrm{mL}$ of added RSS conferred the highest VSL and linearity index values $(P<0.05)$. The velocity of the average path value of the treatment groups containing $0.02,0.04,0.06,0.08$, and $0.10 \mathrm{mg} /$ $\mathrm{mL}$ of RSS was higher than that of control group $(P$ $<0.05)$. No significant difference was observed for the ALH value between freezing extenders supplemented with 0.04 and $0.06 \mathrm{mg} / \mathrm{mL}$ of RSS, and for the VCL value between 0.06 and $0.08 \mathrm{mg} / \mathrm{mL}$ concentrations of RSS in extenders $(P>0.05)$. These results indicate that supplementation of $0.06 \mathrm{mg} / \mathrm{mL}$ of RSS in basic extenders significantly improved sperm motility and movement characteristics.

\section{The Physiological Features of Sperm}

The mitochondrial activities of frozen-thawed sperm supplemented with 0.02 and $0.06 \mathrm{mg} / \mathrm{mL}$ of RSS in extenders were higher than those of other groups $(P$ $<0.05)$, and no significant difference was observed between them (Table 2). The extenders supplemented with $0.06 \mathrm{mg} / \mathrm{mL}$ of RSS led to the highest sperm membrane integrity percentage compared with other treatment groups and control levels $(P<0.05)$. No significant differences for acrosome integrity percentages were observed among the extenders with 0.02, 0.04, and $0.06 \mathrm{mg} / \mathrm{mL}$ of added RSS $(P>0.05)$, and acrosome integrity percentage of these groups were higher than that of the other groups $(P<0.05)$. These results imply that extenders with a concentration of $0.06 \mathrm{mg} /$ $\mathrm{mL}$ of RSS significantly improved sperm mitochondrial activity, membrane integrity, and acrosome integrity.

Figure 1 shows sperm with FITC-PNA staining under a fluorescence microscope. The whole acrosome with strong green fluorescence was not damaged.

Table 1. Linear motile sperm (LM), curvilinear line velocity (VCL), straight line velocity (VSL), linearity index (LIN), amplitude of the lateral head displacement (ALH), and velocity of the average path (VAP) values in frozen-thawed bull spermatozoa (mean \pm SEM)

\begin{tabular}{|c|c|c|c|c|c|c|}
\hline Item & Control & \multicolumn{5}{|c|}{ Rhodiola sachalinensis saccharide $(\mathrm{mg} / \mathrm{mL})$} \\
\hline LM (\%) & $38.2 \pm 0.9^{c}$ & $39.0 \pm 1.0^{\mathrm{c}}$ & $52.2 \pm 0.8^{\mathrm{a}}$ & $53.1 \pm 0.5^{\mathrm{a}}$ & $44.1 \pm 0.5^{\mathrm{b}}$ & $37.9 \pm 0.4^{\mathrm{c}}$ \\
\hline VSL $(\mu \mathrm{m} / \mathrm{s})$ & $30.6 \pm 0.9^{\mathrm{b}}$ & $30.2 \pm 1.1^{\mathrm{b}}$ & $30.9 \pm 1.2^{\mathrm{b}}$ & $33.8 \pm 0.9^{\mathrm{a}}$ & $30.9 \pm 1.1^{\mathrm{b}}$ & $30.2 \pm 0.8^{\mathrm{b}}$ \\
\hline $\operatorname{LIN}(\%)$ & $73.8 \pm 1.1^{\mathrm{b}}$ & $73.4 \pm 1.0^{\mathrm{b}}$ & $74.4 \pm 1.0^{\mathrm{b}}$ & $77.5 \pm 0.9^{\mathrm{a}}$ & $73.5 \pm 1.0^{\mathrm{b}}$ & $73.6 \pm 0.5^{\mathrm{b}}$ \\
\hline $\mathrm{ALH}(\mu \mathrm{m})$ & $1.0 \pm 0.1^{\mathrm{b}}$ & $1.2 \pm 0.09^{\mathrm{b}}$ & $1.5 \pm 0.7^{\mathrm{a}}$ & $1.5 \pm 0.09^{\mathrm{a}}$ & $1.1 \pm 0.05^{\mathrm{b}}$ & $1.1 \pm 0.01^{\mathrm{b}}$ \\
\hline
\end{tabular}

\footnotetext{
${ }^{a-c}$ Values within a row with different superscript letters are significantly different $(P<0.05)$.
} 
Table 2. Percentages of mitochondrial activity, membrane integrity, and acrosome integrity in frozen-thawed bull spermatozoa (mean \pm SEM)

\begin{tabular}{|c|c|c|c|c|c|c|}
\hline Item & Control & \multicolumn{5}{|c|}{ Rhodiola sachalinensis saccharide $(\mathrm{mg} / \mathrm{mL})$} \\
\hline $\begin{array}{l}\text { Mitochondrial activity (\%) } \\
\text { Membrane integrity (\%) } \\
\text { Acrosome integrity (\%) }\end{array}$ & $\begin{array}{l}30.42 \pm 0.05^{\mathrm{d}} \\
32.59 \pm 1.18^{\mathrm{d}} \\
83.01 \pm 0.51^{\mathrm{b}}\end{array}$ & $\begin{array}{l}42.12 \pm 1.25^{\mathrm{a}} \\
41.16 \pm 0.24^{\mathrm{c}} \\
83.69 \pm 0.56^{\mathrm{ab}}\end{array}$ & $\begin{array}{l}40.36 \pm 0.01^{\mathrm{b}} \\
43.98 \pm 0.79^{\mathrm{b}} \\
83.88 \pm 0.08^{\mathrm{a}}\end{array}$ & $\begin{array}{l}42.78 \pm 0.41^{\mathrm{a}} \\
46.45 \pm 0.34^{\mathrm{a}} \\
84.23 \pm 0.24^{\mathrm{a}}\end{array}$ & $\begin{array}{l}34.66 \pm 0.19^{\mathrm{c}} \\
39.12 \pm 1.22^{\mathrm{c}} \\
82.65 \pm 1.33^{\mathrm{b}}\end{array}$ & $\begin{array}{l}30.12 \pm 0.33^{\mathrm{d}} \\
32.78 \pm 0.43^{\mathrm{d}} \\
82.38 \pm 0.43^{\mathrm{b}}\end{array}$ \\
\hline
\end{tabular}

${ }^{\mathrm{a}-\mathrm{d}}$ Values within a row with different superscript letters are significantly different $(P<0.05)$.

\section{Assays of SOD, LDH, and GOT}

The SOD, LDH, and GOT activities were all reduced after cryopreservation of fresh semen (Table 3). Rhodiola sachalinensis saccharide added in the basic extenders showed some cryoprotective capacity (Table 4). The extenders containing 0.06 and $0.08 \mathrm{mg} / \mathrm{mL}$ of RSS significantly improved SOD and LDH activities after freeze-thawing compared with other treatment groups and the control group $(P<0.05)$. However, no significant difference was observed for SOD and LDH activities of sperm samples with 0.06 or $0.08 \mathrm{mg} / \mathrm{mL}$ concentrations of RSS in the basic extenders $(P>0.05)$. In addition, the GOT activities of frozen-thawed sperm in the extenders containing 0.04 and $0.06 \mathrm{mg} / \mathrm{mL}$ of RSS were higher than other concentrations used $(P<$ 0.05). To summarize, the activities of SOD, LDH, and GOT were all decreased during the process of freezing and thawing, and supplementation of $0.06 \mathrm{mg} / \mathrm{mL}$ of RSS in basic extenders significantly improved SOD, $\mathrm{LDH}$, and GOT activities in cryopreserved sperm.

\section{DISCUSSION}

Our results in the present experiments showed that RSS can keep the motility of bull sperm after freezethaw with its addition into the extender, and the percentages of mitochondrial activity and membrane and acrosome integrity of frozen-thawed sperm were also higher in the groups with RSS treatment than that of the control group. Therefore, RSS can protect bull sperm from the damage of freeze-thawing because of the increase of antioxidase activity. Many papers have confirmed that RSS, which is extracted from Rhodiola, as well as Rhodiola water decoction, and rhodioloside have biological functions of antioxidation and antiapop- tosis (Tian and Wang, 2006), and abilities of eliminating $\mathrm{O}^{2-}$ and restraining lipid peroxidation (LPO; Zhang and Liu, 2005). Related studies show that RSS also has functions of antistatic fatigue and dynamic fatigue (Chen et al., 2000). These characteristics may help to reduce damage of sperm during the process of freeze-thawing.

Rhodiola sachalinensis saccharide is one of traditional Chinese medicine herbs that have shown functions of sperm cryopreservation. For instance, Gynostemma pentaphyllum polysaccharide and Lycium barbarum polysaccharide were reported to have cryoprotective effects on sperm (He et al., 2009; Hu et al., 2009). Our results showed that the motive parameters of sperm were protected well with RSS treatments. The percentages of linear motile sperm were higher with the extenders containing 0.04 and $0.06 \mathrm{mg} / \mathrm{mL}$ concentrations of RSS than other concentrations. And $0.06 \mathrm{mg} / \mathrm{mL}$ of RSS added to the extenders almost led to the highest mitochondrial activity and membrane integrity percentage. It was investigated that Rhodiola sacra aqueous extract (RSEA) supplementation in extenders with or without glycerol had significant effects on sperm quality in boar spermatozoa (Zhao et al., 2009). It indicated that the optimal concentration range of RSEA was from 0.04 to $0.08 \mathrm{mg} / \mathrm{mL}$ in glycerol-free and glycerol-containing extenders, and the effects of RSEA on boar semen quality were more evident in the glycerol-free extenders. Our results were consistent with outcomes of Zhao et al. (2009) in the distinct effect of extracts from Rhodiola roots on spermatozoa, but more research is needed on whether the bull semen quality in RSS extenders without glycerol would also be better than those with glycerol.

Reactive oxygen species (ROS), which are produced by way of sperm respiratory metabolism, are harm-

Table 3. Superoxide dismutase (SOD), lactate dehydrogenase (LDH), and glutamic oxaloacetic transaminase (GOT) activity in frozen-thawed bull spermatozoa (mean \pm SEM)

\begin{tabular}{lccl}
\hline Item & SOD $(\mathrm{U} / \mathrm{mL})$ & LDH $(\mathrm{U} / \mathrm{mL})$ & GOT $(\mathrm{U} / \mathrm{mL})$ \\
\hline Fresh semen & $110.23 \pm 0.18^{\mathrm{a}}$ & $4.37 \pm 0.12^{\mathrm{a}}$ & $67.82 \pm 0.93^{\mathrm{a}}$ \\
Cryopreserved semen & $62.02 \pm 0.02^{\mathrm{b}}$ & $2.32 \pm 0.04^{\mathrm{b}}$ & $34.44 \pm 1.50^{\mathrm{b}}$ \\
\hline
\end{tabular}

${ }^{\mathrm{a}, \mathrm{b}}$ Values within a column with different superscript letters are significantly different $(P<0.05)$. 
Table 4. Effects of different Rhodiola sachalinensis saccharide (RSS) concentrations on superoxide dismutase (SOD), lactate dehydrogenase (LDH), and glutamic oxaloacetic transaminase (GOT) activity in frozen-thawed bull spermatozoa (mean \pm SEM)

\begin{tabular}{|c|c|c|c|c|c|c|}
\hline \multirow[b]{2}{*}{ Item } & \multirow[b]{2}{*}{ Control } & \multicolumn{5}{|c|}{ Rhodiola sachalinensis saccharide $(\mathrm{mg} / \mathrm{mL})$} \\
\hline & & 0.02 & 0.04 & 0.06 & 0.08 & 0.10 \\
\hline $\begin{array}{l}\text { SOD }(\mathrm{U} / \mathrm{mL}) \\
\text { LDH }(\mathrm{U} / \mathrm{mL}) \\
\text { GOT }(\mathrm{U} / \mathrm{mL})\end{array}$ & $\begin{array}{r}62.02 \pm 0.02^{\mathrm{c}} \\
2.32 \pm 0.04^{\mathrm{c}} \\
34.44 \pm 1.50^{\mathrm{c}}\end{array}$ & $\begin{array}{r}65.44 \pm 2.01^{\mathrm{c}} \\
2.41 \pm 0.01^{\mathrm{c}} \\
35.61 \pm 0.74^{\mathrm{b}}\end{array}$ & $\begin{array}{r}69.87 \pm 0.08^{\mathrm{b}} \\
2.92 \pm 0.03^{\mathrm{b}} \\
36.99 \pm 1.32^{\mathrm{ab}}\end{array}$ & $\begin{array}{r}73.80 \pm 1.92^{\mathrm{a}} \\
3.55 \pm 0.05^{\mathrm{a}} \\
37.84 \pm 0.90^{\mathrm{a}}\end{array}$ & $\begin{aligned} 73.01 & \pm 0.01^{\mathrm{a}} \\
3.41 & \pm 0.01^{\mathrm{a}} \\
36.28 & \pm 1.20^{\mathrm{b}}\end{aligned}$ & $\begin{aligned} 71.19 & \pm 0.74^{\mathrm{ab}} \\
3.01 & \pm 0.06^{\mathrm{b}} \\
35.53 & \pm 0.82^{\mathrm{b}}\end{aligned}$ \\
\hline
\end{tabular}

${ }^{\mathrm{a}-\mathrm{C}}$ Values within a row with different superscript letters are significantly different $(P<0.05)$.

ful to sperm. The excessive ROS (mainly $\mathrm{O}^{2-}, \mathrm{H}_{2} \mathrm{O}_{2}$, and $-\mathrm{OH})$ are react with peroxidation of membrane phospholipids, and they lead to a decrease in sperm motility and impairment of sperm membranes (Alvarez and Storey, 1992; Askari et al., 1994). Moreover, glutathione peroxidase and other antioxidant enzymes are lost during cryopreservation of sperm (Gadea et al., 2004), which causes the loss of the equilibrium between oxidation and antioxidation and affects the physiological functions of sperm. Cryopreservation can affect the lipid organization and chemical compositions of semen

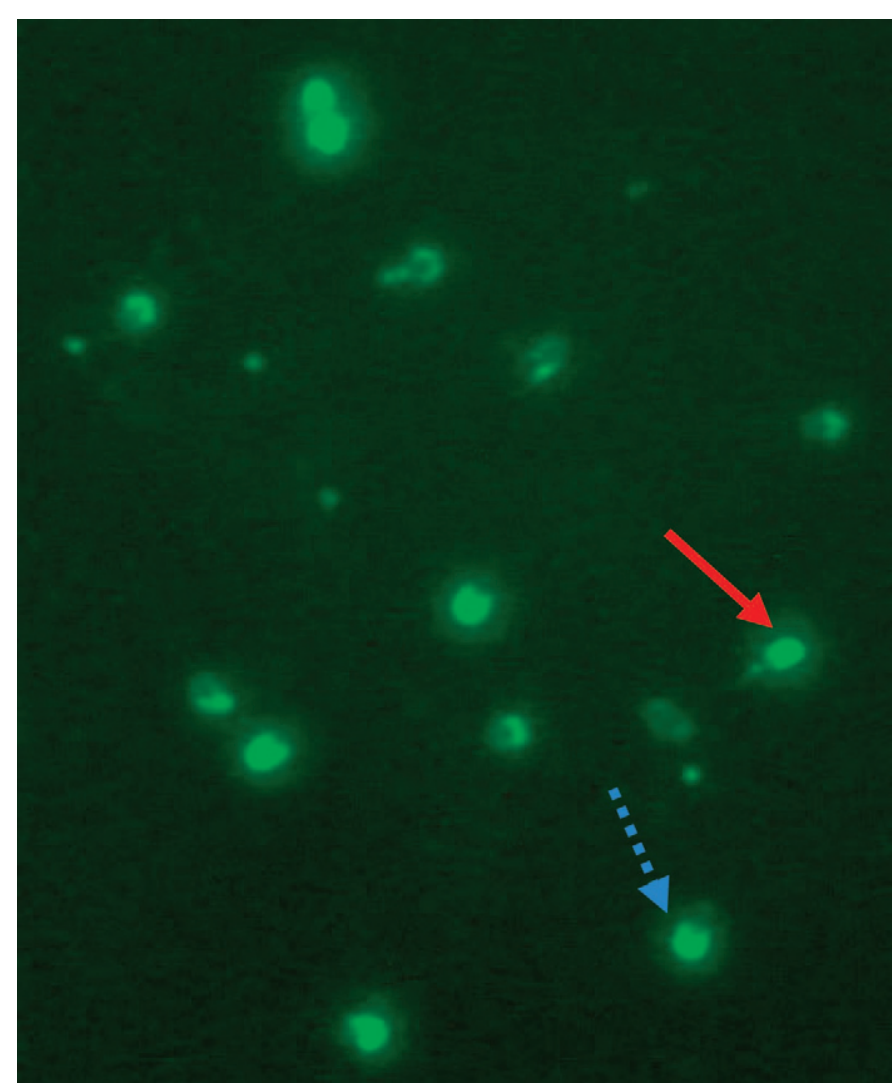

Figure 1. Fluorescent patterns of bull spermatozoa stained with fluorescein isothiocyanate-conjugated peanut agglutinin (FITC-PNA; $400 \times$ ). Spermatozoa observed were frozen-thawed in extender without Rhodiola sachalinensis saccharide (RSS). Red (solid gray) arrow = sperm with intact acrosome; blue (dotted arrow) $=$ sperm with partially damaged acrosome. Color version available in the online PDF. membranes (Amann and Pickett, 1987). Exposed to a cryogenic environment, the plasma membrane of sperm is injured by physical and chemical factors, including thermal, oxidative, and osmotic stress; alterations of the transitions of the lipid phases; and increases in LPO of the plasma membrane induced by ROS (Alvarez and Storey, 1992; Medeiros et al., 2002). The antioxidant system of SOD, catalase, and glutathione peroxidase is considered as a defense infrastructure against LPO in spermatozoa, and it is important to sustain sperm motility and viability (Bilodeau et al., 2001). Superoxide dismutase is a well-known enzymatic biological antioxidant. It controls oxidative stress in mammalian sperm through scavenging ROS (Fridovich I., 1985). In addition to enzymes related to oxidation or ROS, LDH and GOT also play a momentous role in sperm viability, motility, and membrane stability. The bulk of GOT are found on or in the sperm cells initially but not in the seminal plasma, and the measurement of cellular impairment can be detected using GOT release (Pace and Graham, 1970). Lactate dehydrogenase is located in the medial and posterior part of the mitochondrial sheath within sperm and is known as one of the important glycolytic enzymes (Afromeev and Tkachenko, 1999). The glycolysis provides ATP for respiration in sperm, so LDH is critical to sperm energy metabolism (Comhaire et al., 1987).

The activities of LDH and GOT (aspartate transaminase) were reported to be used as markers for sperm freezability and fertility (Dhami and Kodagali, 1990). A decrease in SOD, LDH, and GOT activities in cryopreserved sperm meant the impairment of the sperm plasma membrane and a reduction in sperm motility and viability. The SOD, LDH, and GOT were speculated to pass through the sperm cytomembrane out of the sperm cells because of the membrane damage. The results of our experiment indicated that all the activities of SOD, LDH, and GOT decreased in frozenthawed bull sperm. This phenomenon was also observed in cooled sperm; the SOD activity was reduced in bovine spermatozoa but increased in seminal plasma after sperm was stored at refrigeration temperature over 72 h (Nair et al., 2006). Their outcome stated clearly that SOD activities in spermatozoa were negatively corre- 
lated with LPO of the spermatozoa plasma membrane, and positively correlated with motility and viability of bull spermatozoa. Other studies reported that the activity of GOT and LDH enzymes in the postthaw buffalo seminal plasma increased, whereas the sperm motility decreased (Dhami and Kodagali, 1990), and in China Holstein dairy cows, these enzymes of the frozenthawed sperm showed depressed activities (Zhou et al., 1985).

The current study also indicated that supplementation of RSS improved the activities of SOD, LDH, and GOT in bull sperm, and that the optimal concentration of RSS was $0.06 \mathrm{mg} / \mathrm{mL}$. It had never been studied, as far as we know, until now. Based on the positive correlation between concentrations of RSS and SOD, $\mathrm{LDH}$, and GOT activities, it was deduced that RSS effectively eliminated ROS in excess and blocked LPO to protect sperm. Small decreases were observed at RSS concentrations of 0.08 and $0.10 \mathrm{mg} / \mathrm{mL}$ among the values of the sperm motion parameters, the sperm physiological parameters, and enzyme activities tested in this study. The explanation may be that overly high concentrations of RSS could cripple the sperm motility by over-restraining ROS during the freeze-thaw process (de Lamirande and O'Flaherty, 2008).

\section{CONCLUSIONS}

Based on this study, when RSS was added to extenders used to store bovine semen, it conferred a greater cryoprotective capacity to bull cryopreserved spermatozoa. As a result, the RSS seems to improve the freezability of bull spermatozoa, and the optimal concentration of RSS added into basic extenders was $0.06 \mathrm{mg} / \mathrm{mL}$. The present study was the first evaluation of the effects of RSS on cryopreserved bull semen and SOD, LDH, and GOT activities in bull sperm. Further research is required to obtain more information about the cryoprotective mechanisms of RSS and the detailed membrane damage system in cryopreserved bull spermatozoa.

\section{ACKNOWLEDGMENTS}

This research was supported by the 12th Five-Year Plan Rural Areas State Science and Technology Support Projects (2011BAD19B04-3) and Science and Technology Innovation Project of Shaanxi Province (2011KTCL02-11).

\section{REFERENCES}

Abidov, M., F. Crendal, S. Grachev, R. Seifulla, and T. Ziegenfuss. 2003. Effect of extracts from Rhodiola rosea and Rhodiola crenulata
(Crassulaceae) roots on ATP content in mitochondria of skeletal muscles. Bull. Exp. Biol. Med. 136:585-587.

Aboagla, E. M. E., and T. Terada. 2003. Trehalose-enhanced fluidity of the goat sperm membrane and its protection during freezing. Biol. Reprod. 69:1245-1250.

Aboagla, E. M. E., and T. Terada. 2004. Effects of the supplementation of trehalose extender containing egg yolk with sodium dodecyl sulfate on the freezability of goat spermatozoa. Theriogenology 62:809-818.

Afromeev, V. I., and V. N. Tkachenko. 1999. Change in the percent of lactate dehydrogenase isoenzyme level in testes of animals exposed to superhigh frequency radiation. Biofizika 44:931-932.

Alvarez, J. G., and B. T. Storey. 1992. Evidence for increased lipid peroxidative damage and loss of superoxide dismutase activity as a mode of sublethal cryodamage to human sperm during cryopreservation. J. Androl. 13:232-241.

Amann, R. P., and B. W. Pickett. 1987. Principles of cryopreservation and a review of cryopreservation of stallion spermatozoa. J. Equine Vet. Sci. 7:145-173.

Askari, H. A., J. H. Check, N. Peymer, and A. Bollendorf. 1994. Effect of natural antioxidants tocopherol and ascorbic acids in maintenance of sperm activity during freeze-thaw process. Arch. Androl. $33: 11-15$.

Bilodeau, J. F., S. Blanchette, I. C. Gagnon, and M. A. Sirard. 2001. Thiols prevent $\mathrm{H}_{2} \mathrm{O}_{2}$-mediated loss of sperm motility in cryopreserved bull semen. Theriogenology 56:275-286.

Blanco, A., C. Burgos, N. M. Gerez de Burgos, and E. E. Montamat. 1976. Properties of the testicular lactate dehydrogenase isoenzyme. Biochem. J. 153:165-172.

Borque, C., and A. Ayllón. 1996. Aspartate aminotransferase in ejaculates of Manchego and Merino rams after minimal and maximal sperm damage. Theriogenology 46:1017-1025.

Chanapiwat, P., K. Kaeoket, and P. Tummaruk. 2012. Cryopreservation of boar semen by egg yolk-based extenders containing lactose or fructose is better than sorbitol. J. Vet. Med. Sci. 74:351-354.

Chen, C. W., L. C. Zhang, Z. X. Ji, and X. H. Li. 2000. Research on the use of Rhodiola sachalinensis A. Bor. in soft drinks. Beverage Industry $3: 29-30$.

Comhaire, F. H., L. Vermeulen, and F. Schoonjans. 1987. Reassessment of the accuracy of traditional sperm characteristics and adenosine triphosphate (ATP) in estimating the fertilizing potential of human semen in vivo. Int. J. Androl. 10:653-662.

Corcuera, B. D., P. Marigorta, A. Sagüés, F. Saiz-Cidoncha, and J. F. Pérez-Gutiérrez. 2007. Effect of lactose and glycerol on the motility, normal apical ridge, chromatin condensation and chromatin stability of frozen boar spermatozoa. Theriogenology 67:11501157.

de Lamirande, E., and C. O'Flaherty. 2008. Sperm activation: Role of reactive oxygen species and kinases. Biochim. Biophys. Acta 1784:106-115.

Dhami, A. J., and S. B. Kodagali. 1990. Freezability, enzyme leakage and fertility of buffalo spermatozoa in relation to the quality of semen ejaculates and extenders. Theriogenology 34:853-863.

Eriksson, B. M., H. Petersson, and H. Rodriguez-Martinez. 2002. Field fertility with exported boar semen frozen in the new flatpack container. Theriogenology 58:1065-1079.

Flohé, L., and F. Otting. 1984. Superoxide dismutase assays. Methods Enzymol. 105:93-104.

Fridovich, I. 1985. Superoxide dismutase, regularities and irregularities. Harvey Lect. 79:51-75.

Gadea, J., E. Sellés, M. A. Marco, P. Coy, C. Matás, R. Romar, and S. Ruiz. 2004. Decrease in glutathione content in boar sperm after cryopreservation: Effect of the addition of reduced glutathione to the freezing and thawing extenders. Theriogenology 62:690-701.

Garner, D. L. 1991. Artificial insemination. Pages 251-278 in Reproduction in Domestic Animals. 4th ed. P. T. Cupps, ed. Academic Press, San Diego, CA.

Garner, D. L., C. A. Thomas, H. W. Joerg, J. M. DeJarnette, and C. E. Marshall. 1997. Fluorometric assessments of mitochondrial function and viability in cryopreserved bull spermatozoa. Biol. Reprod. 57:1401-1406. 
Gómez-Fernández, J., E. Gómez-Izquierdo, C. Tomás, E. Mocé, and E. de Mercado. 2012. Effect of different monosaccharides and disaccharides on boar sperm quality after cryopreservation. Anim. Reprod. Sci. 133:109-116.

Gonda, T., and T. Sei. 2005. The inhibitory growth mechanism of saccharides on the growth of ice crystals from aqueous solutions. Prog. Cryst. Growth Charact. Mater. 51:70-80.

Gong, G. M., H. T. Wang, and N. Han. 2005. Extraction of polysaccharide from Rhodiola with ultrasonic wave. Food Sci., China 26:127-130.

He, T., Q. W. Li, and B. Wang. 2009. The effects of Lycium barbarum polysaccharide on cryopreserved boar spermatozoa. J. Shaanxi Agric. Sci. 4:3-5.

Hu, J. H., Q. W. Li, G. Li, L. Q. Wang, and Z. L. Jiang. 2006. The cryoprotective effect on frozen-thawed boar semen of egg yolk low density lipoproteins. Asian-australas. J. Anim. Sci. 19:486-490.

Hu, J. H., Q. W. Li, T. Zhang, and Z. L. Jiang. 2009. Effect of Gynostemma pentaphyllum polysaccharide on boar spermatozoa quality following freezing-thawing. Cryobiology 59:244-249.

Jeyendran, R. S., H. H. Van der Ven, M. Perez-Pelaez, B. G. Crabo, and L. J. D. Zaneveld. 1984. Development of an assay to assess the functional integrity of the human sperm membrane and its relationship to other semen characteristics. J. Reprod. Fertil. 70:219-228.

Johnson, L. A., K. F. Weitze, P. Fiser, and W. M. C. Maxwell. 2000. Storage of boar semen. Anim. Reprod. Sci. 62:143-172.

Katkov, I. I., N. Katkova, J. K. Critser, and P. Mazur. 1998. Mouse spermatozoa in high concentrations of glycerol: chemical toxicity vs osmotic shock at normal and reduced oxygen concentrations. Cryobiology 37:325-338.

Kumar, S., J. D. Millar, and P. F. Watson. 2003. The effect of cooling rate on the survival of cryopreserved bull, ram, and boar spermatozoa: A comparison of two controlled-rate cooling machines. Cryobiology 46:246-253.

Li, Y.-H., K.-J. Cai, A. Kovacs, and W.-Z. Ji. 2005. Effects of various extenders and permeating cryoprotectants on cryopreservation of cynomolgus monkey (Macaca fascicularis) spermatozoa. J. Androl. 26:387-395.

Liu, Z., R. H. Foote, and C. C. Brockett. 1998. Survival of bull sperm frozen at different rates in media varying in osmolarity. Cryobiology $37: 219-230$.

Maxwell, W. M. C., G. Evans, S. T. Mortimer, L. Gillan, E. S. Gellatly, and C. A. McPhie. 1999. Normal fertility in ewes after cervical insemination with frozen-thawed spermatozoa supplemented with seminal plasma. Reprod. Fertil. Dev. 11:123-126.

Medeiros, C. M. O., F. Forell, A. T. D. Oliveira, and J. L. Rodrigues. 2002. Current status of sperm cryopreservation: Why isn't it better? Theriogenology 57:327-344.

Nair, S. J., A. S. Brar, C. S. Ahuja, S. P. S. Sangha, and K. C. Chaudhary. 2006. A comparative study on lipid peroxidation, activities of antioxidant enzymes and viability of cattle and buffalo bull spermatozoa during storage at refrigeration temperature. Anim. Reprod. Sci. 96:21-29.

Osinowo, O. A., J. O. Bale, E. O. Oyedipe, and L. O. Eduvie. 1982 Motility and eosin uptake of formaldehyde-treated ram spermatozoa. J. Reprod. Fertil. 65:389-394.

Pace, M. M., and E. F. Graham. 1970. The release of glutamic oxaloacetic transaminase from bovine spermatozoa as a test method of assessing semen quality and fertility. Biol. Reprod. 3:140-146.

Parrish, J. J., A. Krogenaes, and J. L. Susko-Parrish. 1995. Effect of bovine sperm separation by either swim-up or Percoll method on success of in vitro fertilization and early embryonic development. Theriogenology 44:859-869.

Salamon, S., and W. M. C. Maxwell. 2000. Storage of ram semen. Anim. Reprod. Sci. 62:77-111.

Schäfer-Somi, S., S. Kluger, E. Knapp, D. Klein, and C. Aurich. 2006. Effects of semen extender and semen processing on motility and viability of frozen-thawed dog spermatozoa. Theriogenology $66: 173-182$

Song, X.-W., L. Ren, Y.-P. Han, Z.-B. Cui, and J.-K. Huang. 2008. Purification and composition analysis of polysaccharide RCPS from Rhodiola crenulata. Guang $\mathrm{Pu}$ Xue Yu Guang Pu Fen Xi 28:642-644.

Sztein, J. M., K. Noble, J. S. Farley, and L. E. Mobraaten. 2001 Comparison of permeating and nonpermeating cryoprotectants for mouse sperm cryopreservation. Cryobiology 42:28-39.

Tian, M., and M. Wang. 2006. Studies on extraction, isolation and composition of Lycium barbarum polysaccharides. Zhongguo Zhong Yao Za Zhi 31:1603-1607.

Woelders, H., A. Matthijs, and B. Engel. 1997. Effects of trehalose and sucrose, osmolality of the freezing medium, and cooling rate on viability and intactness of bull sperm after freezing and thawing. Cryobiology 35:93-105.

Yousef, G. G., M. H. Grace, D. M. Cheng, I. V. Belolipov, I. Raskin, and M. A. Lila. 2006. Comparative phytochemical characterization of three Rhodiola species. Phytochemistry 67:2380-2391.

Zhang, S., H. Bi, and C. Liu. 2007. Extraction of bio-active components from Rhodiola sachalinensis under ultrahigh hydrostatic pressure. Separ. Purif. Technol. 57:277-282.

Zhang, Y., and Y. Liu. 2005. Study on effects of salidroside on lipid peroxidation on oxidative stress in rat hepatic stellate cells. Zhong Yao Cai 28:794-796.

Zhao, H.-W., Q.-W. Li, G.-Z. Ning, Z.-S. Han, Z.-L. Jiang, and Y.-F Duan. 2009. Rhodiola sacra aqueous extract (RSAE) improves biochemical and sperm characteristics in cryopreserved boar semen. Theriogenology 71:849-857.

Zhou, J. Y., S. L. Niu, Z. Y. Feng, Q. Z. Lin, and S. H. Jin. 1985. Changes of sperm acrosomes and enzymes in seminal plasma of Chinese-Holstein dairy cows during freezing process. China Dairy Cattle 3:5-9. 